\title{
Smoking Restores Impaired LTD-Like Plasticity in Schizophrenia: a Transcranial Direct Current Stimulation Study
}

\author{
Wolfgang Strube', Tilmann Bunse', Michael A Nitsche ${ }^{2}$, Thomas Wobrock ${ }^{3,4}$, Richard Aborowa ${ }^{4}$, \\ Kristina Misewitsch ${ }^{4}$, Maximiliane Herrmann ${ }^{4}$, Peter Falkai' and Alkomiet Hasan*,I \\ 'Department of Psychiatry and Psychotherapy, Ludwig Maximilian University, Munich, Germany; '2Department of Clinical Neurophysiology, \\ University of Goettingen, Goettingen, Germany; ${ }^{3}$ Centre of Mental Health, Darmstadt-Dieburg Clinics, Darmstadt, Germany; ${ }^{4}$ Department of \\ Psychiatry and Psychotherapy, University of Goettingen, Goettingen, Germany
}

Impaired neuroplastic responses following noninvasive brain stimulation have been reported repeatedly in schizophrenia patients. These findings have been associated with deficits in GABAergic, glutamatergic, and cholinergic neurotransmission. Although various neurophysiological studies have indicated a relationship between nicotine and neuroplasticity in healthy individuals, the present study is the first investigation into the impact of nicotine on LTD-like plasticity in patients with schizophrenia. Cortical excitability and cortical plasticity were explored in 30 schizophrenia patients ( 17 smoker, 13 nonsmoker) and 45 healthy controls ( 13 smoker, 32 nonsmoker) by using single-pulse transcranial magnetic stimulation (TMS) before and following cathodal transcranial direct current stimulation (tDCS) applied to the left primary motor cortex. Our analysis revealed abolished LTD-like plasticity in nonsmoking schizophrenia patients. However, these plasticity deficits were not present in smoking schizophrenia patients. In healthy controls, significant MEP reductions following cathodal tDCS were observed in nonsmoking individuals, but only trend-level reductions in smokers. In smoking schizophrenia patients, the severity of negative symptoms correlated positively with reduced neuroplasticity, whereas nonsmoking patients displayed the opposite effect. Taken together, the data of our study support the notion of an association between chronic smoking and the restitution of impaired LTD-like plasticity in schizophrenia patients. Although replication and further research are needed to better understand this relationship, our findings indicate that nicotine intake might stabilize the impaired inhibition-facilitation balance in the schizophrenic brain through a complex interaction between cortical plasticity, and GABAergic and cholinergic neurotransmission, and might explain the reduced prevalence of negative symptoms in this population.

Neuropsychopharmacology (20I5) 40, 822-830; doi:I 0.I038/npp.2014.275; published online 5 November 20I4

\section{INTRODUCTION}

Severe mental illness is highly associated with an increased prevalence of cigarette smoking and tobacco dependency. Specifically, elevated average smoking rates $>60 \%$ have been reported in patients suffering from schizophrenia (Dome et al, 2010). Different theories implicate nicotine intake via smoking as a compensatory strategy used by schizophrenia patients to ameliorate their symptoms (D'Souza and Markou, 2012).

Indicating complex alterations of the cholinergic system, rodent schizophrenia models revealed reduced $\alpha_{7}$-nAChreceptor (nAChR) expression levels and an increased release of acetylcholine (Hashimoto et al, 2008a; Nelson et al, 2002).

*Correspondence: Dr A Hasan, Department of Psychiatry and Psychotherapy, Ludwig Maximilian University, Nussbaumstrasse 7, D- 80336 Munich, Germany, Tel: +49 0894400 555। I, Fax: +40 0894400 55530, E-mail: Alkomiet.Hasan@med.uni-muenchen.de Received 12 July 2014; revised 24 September 2014; accepted 5 October 2014; accepted article preview online 13 October 2014
Accordingly, postmortem studies and pharmacological challenges have reported further evidence of decreased expression levels of $\alpha_{7}$-nAChR in patients with schizophrenia (D'Souza and Markou, 2012; Martin-Ruiz et al, 2003). At the behavioral level, the stimulation of $\alpha_{7}-\mathrm{nAChR}$ through nicotine intake has been demonstrated to improve working memory and selective attention deficits, and to enhance thalamocortical functional connectivity in schizophrenia patients (Jacobsen et al, 2004; Sacco et al, 2005). Taken together, an illness-related dysfunction of corticosubcortical cholinergic networks in patients with schizophrenia, involving a complex interplay of cholinergic activation and neuroplasticity, can be assumed (D'Souza and Markou, 2012).

The role of the most prominent nAChR agonist, nicotine, for neuroplasticity is evident from animal research indicating a prominent influence on plasticity functions (Levin et al, 2006), eg, by showing nicotine-dependent facilitation of long-term potentiation (LTP) (Huerta and Lisman, 1995), a reversal of stress-induced long-term-depression (LTD) deficits (Aleisa et al, 2006), and the involvement of different 
nAchR subtypes in the induction of hippocampal LTD (Nakauchi and Sumikawa, 2014). Although most investigations of the nicotinergic impact on neuroplasticity are restricted to animal studies, neurophysiological studies using transcranial direct current stimulation (tDCS) have recently given insight into the potential impact of nicotine on neuroplasticity at the system level of the human cortex. Cathodal tDCS has been demonstrated to induce longlasting and polarity-dependent changes in motor-cortical excitability, leading to the activation of physiological mechanisms related to molecular long-term-depression (LTD) (Nitsche et al, 2008). Studies applying cathodal tDCS in healthy nonsmokers for plasticity induction showed either a reduction or even an abolishment of LTD-like plasticity following prolonged and fast-acting nicotine application (Grundey et al, 2012a; Thirugnanasambandam et al, 2011). Further investigations on chronic smokers during nicotine withdrawal revealed unaffected LTD-like plasticity following cathodal tDCS, which was abolished following nicotine reapplication (Grundey et al, 2012b). Moreover, physiological investigations with transcranial magnetic stimulation (TMS) indicate that chronic smoking tilts the balance between facilitation and inhibition towards cortical hypoexcitability (Grundey et al, 2013; Lang et al, 2008). In summary, evidence points to a significant modulation of both neuroplasticity and cortical excitability through acute and chronic nicotine intake in healthy smokers and nonsmokers.

Despite the consistent reduction of cortical plasticity in schizophrenia patients following different types of plasticity-inducing non-invasive brain stimulation (Hasan et al, 2013a; Daskalakis et al, 2008a; Frantseva et al, 2008; Oxley et al, 2004) and the association of smoking with functional and symptomatic improvements (D'Souza and Markou, 2012), the impact of nicotine intake via smoking on neuroplasticity in schizophrenia has not yet been investigated. To determine the physiological basis of the association between nicotine and neuroplasticity, we applied LTD-like plasticity-inducing cathodal tDCS to smoking and nonsmoking schizophrenia patients. Given the well-established inhibitory deficit in schizophrenia in various brain regions, including the primary motor cortex (Hashimoto et al, 2008b), the positive impact of nicotine in pronouncing inhibitory functions (Lang et al, 2008), and the concept of smoking-related self-medication, we hypothesized that smoking schizophrenia patients would display fewer deficits in LTD-like plasticity compared with nonsmoking patients. On the basis of previous publications (Grundey et al, 2012b), we further hypothesized that healthy smokers and nonsmokers would display differences in LTD-like plasticity following cathodal tDCS. We also hypothesized that smoking and nonsmoking healthy subjects would show different plasticity responses compared with the respective schizophrenia patients.

\section{MATERIALS AND METHODS}

\section{Subjects}

Thirty schizophrenia patients and 45 healthy controls (aged 18-40 years) from previous studies (Hasan et al, 2012a; Hasan et al, 2013b; Hasan et al, 2012b) were pooled and included in this study, resulting in a total sample size of 75 . The sample recruitment was conducted from 2009 to 2012 . Participants with contraindication to TMS or tDCS were excluded. A clinical psychiatrist and a member of the study group (TW/AH) made an ICD-10 consensus diagnosis. All participants underwent a standardized test of hand preference (Oldfield, 1971), and patients received an assessment of psychopathological symptoms (PANSS), disease severity (CGI), and functioning (GAF). The ethics committee of the UMC Goettingen approved the protocol, which was conducted in accordance with the Declaration of Helsinki.

Sociodemographic variables are presented in Table 1 and concomitant medication is presented in Supplementary Table S1.

\section{TMS}

The complete protocol has been described previously (Hasan et al, 2012b; Hasan et al, 2011). Electromyographic activity (EMG) was recorded by surface electrodes on the right dorsal interosseus muscle in a belly-tendon montage. Raw signals were amplified, bandpass-filtered $(2 \mathrm{~Hz}-10 \mathrm{kHz})$ and digitized using a standard amplifier (Keypoint portable, Medtronic Co., Denmark). TMS was performed over the left primary motor cortex (M1) with a standard 70-mm-TMS figure-of-eight coil and a MagPro-X-100 stimulator (Medtronic, Denmark). Throughout all the experiments, the coil was held tangentially to the head, with the handle pointing backwards and in a $45^{\circ}$ angle lateral to the midline. The stimulation site that produced the largest motor-evoked potential (MEP) at moderately suprathreshold stimulation intensities was marked as the optimal coil position.

\section{tDCS}

Cathodal transcranial direct current stimulation (tDCS) was applied after baseline TMS examination. The tonic electrical field with a stimulation intensity of $1 \mathrm{~mA}$ was induced by a CE-certified stimulator (NeuroConn, Ilmenau, Germany) through saline-soaked rectangular surface sponge electrodes $\left(35 \mathrm{~cm}^{2}\right)$ for a duration of $9 \mathrm{~min}$. These parameters have been consistently shown to be well suited for the induction of cortical excitability alterations lasting for about $1 \mathrm{~h}$ after tDCS (Nitsche et al, 2008). The motor cortex electrode was placed over the left motor cortex (M1) over the location of the optimal TMS coil position for eliciting motor-evoked potentials of the target muscle and the return electrode was placed over the contralateral supraorbital forehead.

\section{Cortical Excitability}

We measured different parameters of cortical excitability (expressed as changes in MEP size) within one experimental session at baseline and one within the first $5 \mathrm{~min}$ following cathodal tDCS. In the case of smokers, data were acquired after at least half an hour of nicotine withdrawal. Resting motor threshold (RMT), expressed as percentage of maximum stimulator output, was defined as the lowest intensity that produced a minimum MEP of $50 \mu \mathrm{V}$ in the relaxed FDI in at least 5 of 10 trials. To monitor after-effects of cathodal tDCS on cortico-spinal excitability, single-pulse 
Table I Demographic and Clinical Characteristics of the Participants

\begin{tabular}{|c|c|c|c|c|c|}
\hline Variable & SZ nonsmoker & SZ smoker & HC nonsmoker & HC smoker & Statistics ( $p$-value) \\
\hline Gender & $4 F, 9 M$ & $5 \mathrm{~F}, 12 \mathrm{M}$ & $13 \mathrm{~F}, 19 \mathrm{M}$ & $5 F, 8 M$ & $0.848^{\mathrm{a}}$ \\
\hline Age & $28.3 \pm 7.9$ & $29.2 \pm 5.4$ & $27.4 \pm 3.6$ & $27.9 \pm 4.6$ & $0.703^{b}$ \\
\hline Handedness & | | R, $2 \mathrm{~L}$ & $14 \mathrm{R}, 3 \mathrm{~L}$ & $30 \mathrm{R}, 2 \mathrm{~L}$ & | | R, $2 \mathrm{~L}$ & $0.616^{\mathrm{a}}$ \\
\hline Fagerstroem-score & - & $5.6 \pm 1.5$ & - & $2.5 \pm 1.9$ & $<0.00 I^{*}, \mathrm{~b}$ \\
\hline \multicolumn{6}{|l|}{ PANSS scores } \\
\hline Negative & $18.7 \pm 2.9$ & $15.4 \pm 5.1$ & - & - & $0.048 *, \mathrm{~b}$ \\
\hline General & $28.1 \pm 4.9$ & $27.8 \pm 8.9$ & - & - & $0.982^{b}$ \\
\hline GAF & $59.2 \pm 12.7$ & $55.4 \pm 10.5$ & - & - & $0.375^{b}$ \\
\hline CGl & $4.3 \pm 1.1$ & $4.4 \pm 0.9$ & - & - & $0.775^{b}$ \\
\hline
\end{tabular}

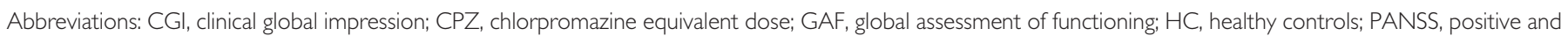
negative syndrome scale; SZ, schizophrenia patients.

Data are presented as mean \pm SD

${ }^{\text {a } C h i-s q u a r e ~ t e s t . ~}$

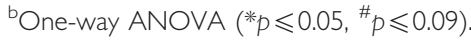

MEPs were recorded from the M1 representation of the right FDI. Stimulation intensity was adjusted at baseline to evoke MEPs of an average size of $1 \mathrm{mV}(\mathrm{S} 1 \mathrm{mV})$, and was then kept unchanged for the assessments after tDCS. CSP duration was recorded as FDI muscle activity under voluntary contraction while stimulating the contralateral M1 using 120\% RMT. The mean CSP duration was then calculated from 10 consecutive trials. The active MEP (aMEP) was measured in 10 trials as peak-to-peak measurement.

\section{Statistics}

For statistical analyses, SPSS 22 was used. Level of significance was set at $\alpha=0.05$. One-way analysis of variance (ANOVA) and Chi-square tests were used to analyze differences between the study groups with regard to demographic variables (age, gender, handedness). Chi-square tests were used to compare the amount of concomitant medication. Age, Fagerstroem scores and baseline excitability parameters were also compared with one-way ANOVAs. Independent $t$-tests were used to compare clinical measures (GAF, CGI, PANSS, duration of illness) in the patient groups. The dependent variables were RMT, $S 1 \mathrm{mV}$, MEP size, CSP, and active MEP (aMEP). Due to the difference in sample sizes, tests for normal distribution were conducted separately for the schizophrenia and healthy control groups. Since the normality assumption was violated for RMT, S1mV, $1 \mathrm{mV}$-MEP, and aMEP in both study groups (Shapiro-Wilk-test, schizophrenia: $p$-values between $<0.001$ and 0.038; healthy controls: $p$-values between $<0.001$ and 0.047), a logarithmic transformation (ln) was applied to the raw values included in further analysis. Repeated-measure-ANOVAs (RM-ANOVA) were computed with 'time' (before $v s$ after tDCS) as withinsubject factor, and 'smoking status' (smoking vs nonsmoking) and 'study group' (schizophrenia patients $v s$ healthy controls) as between-subject factors. In the event of significant interactions, paired $t$-tests were conducted for within-group comparisons and independent $t$-tests in the case of between-group pre-post comparisons (all two-tailed, $p<0.05$, not adjusted for multiple comparisons) to analyze differences between the subgroups. Sphericity was tested with the Mauchly's test and, if necessary, the GreenhouseGeisser correction was used. Pearson correlations between dependent variables and clinical variables were performed.

\section{RESULTS}

\section{Sociodemographic and Clinical Characteristics}

The study groups did not differ significantly with regard to gender distribution $(p=0.848)$, handedness $(p=0.616)$, or age $(p=0.703)$. According to PANSS measures, all schizophrenia patients suffered from moderate positive and negative symptoms and showed similar degrees of illness (CGI) and impairments of global functioning (GAF). Nonsmoking schizophrenia patients presented with significantly more intensive negative symptoms $(p=0.048)$ and showed a trend-level shorter duration of illness $(p=0.095)$. Mean Fagerstroem scores differed between smoking schizophrenia patients $(5.6 \pm 1.5)$ and smoking healthy controls $(2.5 \pm 1.9)(p<0.001)$, indicating a higher level of cigarette consumption and tobacco dependence in schizophrenia patients. Chlorpromazine equivalents $(p=0.836$; Table 1) and the amount of concomitant medication $(p=0.193$; Supplementary Table S1) did not differ between the patient groups.

\section{Cortical Excitability at Baseline}

Four group comparisons of cortical excitability parameters at baseline were conducted using a one-way ANOVA and showed no significant differences across groups but trendwise differences for RMT and S1mV (Table 2). 
Table 2 ANOVA of Cortical Excitability Parameters of the Dependent Variables at Baseline

\begin{tabular}{|c|c|c|c|c|c|}
\hline Variable & SZ nonsmoker & SZ smoker & HC nonsmoker & HC smoker & ANOVA ( $p$-value) \\
\hline RMT (\%) & $59.0 \pm 8.0$ & $52.2 \pm 10.7$ & $50.8 \pm 9.7$ & $49.6 \pm 8.9$ & $0.054^{\#}$ \\
\hline SImV (\%) & $69.1 \pm 9.8$ & $61.0 \pm 12.3$ & $60.0 \pm 13.5$ & $59.0 \pm 8.0$ & $0.070^{\#}$ \\
\hline MEP (mV) & $994.0 \pm 332.4$ & $932.1 \pm 252.5$ & $956.0 \pm 283.7$ & $984.0 \pm 291.3$ & 0.963 \\
\hline CSP (ms) & $149.8 \pm 46.5$ & $140.8 \pm 31.5$ & $\mid 36.1 \pm 32.7$ & $134.4 \pm 36.2$ & 0.768 \\
\hline aMEP (mV) & $4742.1 \pm 2856.9$ & $5512.6 \pm 5058.1$ & $5412.2 \pm 4101.2$ & $4332.4 \pm 3426.3$ & 0.955 \\
\hline
\end{tabular}

Abbreviations: HC, healthy controls; SZ, schizophrenia patients.

Data are presented as mean \pm SD (* $p \leqslant 0.05, \# p \leqslant 0.09)$.

Table 3 RM-ANOVA across all Study Groups Showed a Significant 'Time' and 'Time $\times$ Smoking-state $\times$ Study-group' Interactions for I mV-MEPs (*p $\leqslant 0.05)$

\begin{tabular}{lccc}
\hline RM-ANOVA & $\begin{array}{c}\text { Hypothesis } \\
\text { df, error } \mathbf{d f}\end{array}$ & F-value & p-value \\
\hline I mV-MEP & & & \\
$\quad$ Time & $1,7 \mid$ & 16.792 & $<0.000$ ।* \\
Time $\times$ smoking status & $1,7 \mid$ & 1.730 & 0.193 \\
Time $\times$ group & $1,7 \mid$ & 1.102 & 0.297 \\
Time $\times$ group $\times$ smoking status & I, 7| & 5.479 & $0.022^{*}$ \\
\hline
\end{tabular}

\section{$1 \mathrm{mV}$-MEP before and after Cathodal tDCS}

The RM-ANOVA with the factors 'time', 'smoking status', and 'study group' revealed a significant main effect of 'time' $(F=16.79, p<0.0001)$ and a significant 'time $\times$ smoking status $\times$ study-group' interaction $(F=5.48, \quad p=0.022)$ (Table 3). Paired $t$-tests (within-subject comparisons) to compare MEP sizes before and after cathodal tDCS showed a significant decrease in MEP size in healthy nonsmokers $\left(t_{(31)}=5.501, p<0.0001\right)$ and a trend-level decrease in healthy smokers $\left(t_{(12)}=1.913, p=0.079\right)$. Nonsmoking schizophrenia patients displayed no decrease in MEP size $\left(t_{(12)}=0.294, p=0.774\right)$, whereas smoking schizophrenia patients showed a significant decrease in MEP size following cathodal tDCS $\left(t_{(16)}=2.464, p=0.025\right.$; Figure 1$)$.

Independent-samples $t$-tests (between-subject comparisons) comparing baseline MEP size revealed neither significant differences between healthy nonsmokers and smokers $\left(t_{(43)}=0.342, p=0.773\right)$, nor between schizophrenic nonsmokers and smokers $\left(t_{(28)}=0.385, p=0.702\right)$. Independent-samples $t$-tests also revealed no baseline MEP amplitude differences between healthy and schizophrenic nonsmokers $\left(t_{(43)}=0.257, p=0.798\right)$, as well as no significant differences between healthy and schizophrenic smokers $\left(t_{(28)}=0.496, p=0.624\right)$. After cathodal tDCS, independent-samples $t$-tests showed no significant differences between healthy smokers and nonsmokers $\left(t_{(43)}=1.194, p=0.239\right)$, but did reveal significant differences between schizophrenic smokers and nonsmokers $\left(t_{(28)}=2.074, p=0.047\right)$. Comparisons between healthy and schizophrenic nonsmokers revealed significant differences $\left(t_{(43)}=2.936, p=0.005\right)$, whereas the comparison between

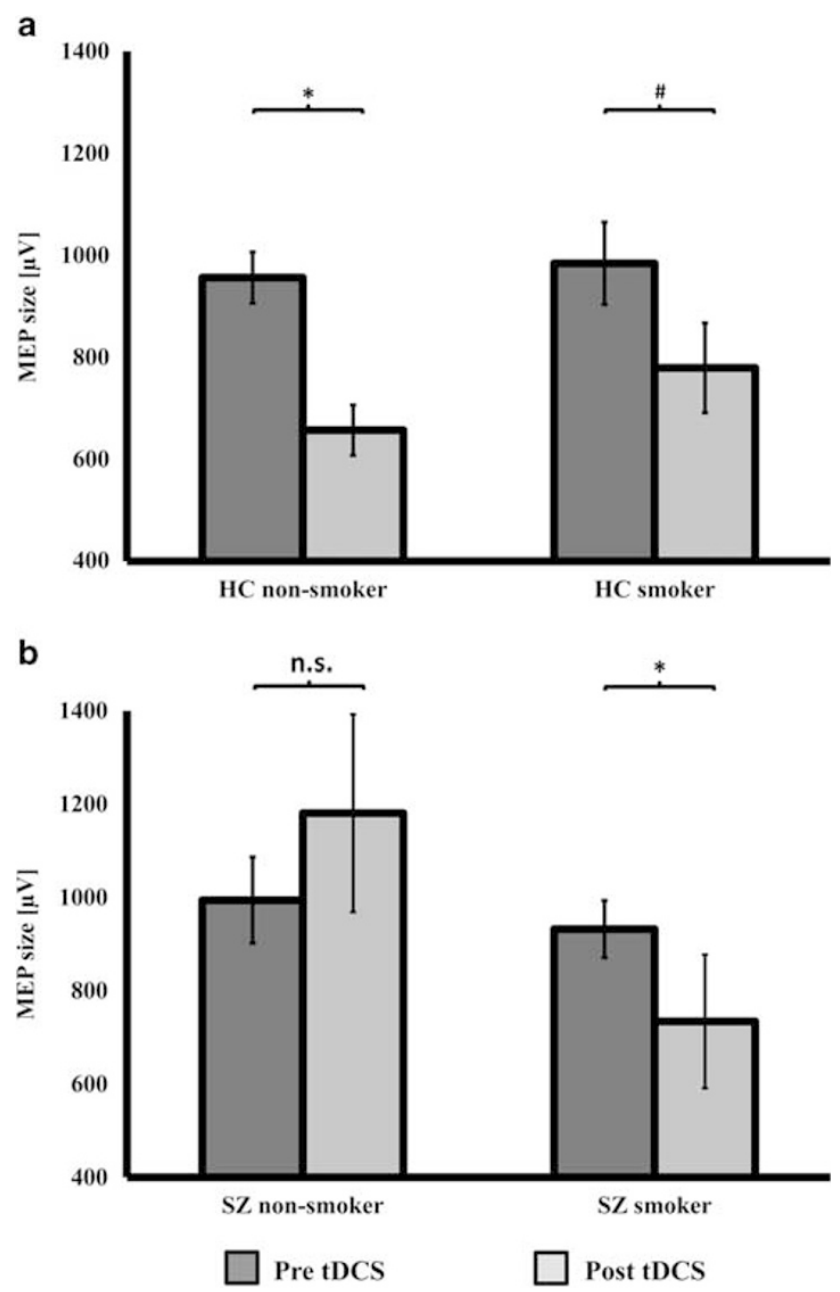

Figure I MEP size before and after cathodal tDCS. (a) Displays MEP sizes before and after cathodal tDCS in healthy nonsmokers and smokers. Dependent $t$-tests showed a significant MEP decrease for healthy nonsmokers $\left(t_{(31)}=5.50 \mathrm{I}, p<0.000 \mathrm{I}\right)$ and a trend-level difference in healthy smokers $\left(t_{(12)}=1.913, p=0.079\right)$. (b) Displays MEP sizes before and after cathodal tDCS in schizophrenia nonsmokers and smokers. A significant MEP reduction was found in the smoking schizophrenia patients $\left(t_{(16)}=2.464, p=0.025\right)$, but not in the nonsmoking schizophrenia patients $\left(t_{(12)}=0.294, p=0.774\right)$. Instead, the latter displayed an opposite numeric increase in I mV-MEP size following LTD-inducing stimulation. Data are presented as mean \pm SEM and graphs show untransformed data.

healthy and schizophrenic smokers showed no significant differences in MEP size $\left(t_{(28)}=0.962, p=0.344\right)$ following cathodal tDCS. 


\section{Other Parameters of Cortical Excitability before and after Cathodal tDCS}

For all other dependent variables, RM-ANOVAs showed no significant main effects or interactions and no further analyses were conducted.

\section{Correlation Analysis}

Pearson correlations conducted in the schizophrenic nonsmoking group between dependent variables clinical variables, and CPZ equivalents revealed significant positive correlations between PANSS $_{\text {Negative }}$ and post RMT values $(p=0.037)$, and between GAF values and post aMEP values $(p=0.039)$. Furthermore, a negative correlation between PANSS $_{\text {Negative }}$ and postMEP sizes $(p=0.019)$ was revealed in this group. Analyses in the schizophrenia smoking group showed a significant positive correlation between PANSS $_{\text {Negative }}$ and post-MEP sizes $(p=0.012$; Figure 2). In healthy smokers, we found a significant negative correlation between Fagerstroem values and CSP duration $(p=0.009)$, and in schizophrenic smokers, positive correlations between Fagerstroem values and pretDCS $\operatorname{aMEP}(p=0.020)$, as well as post-tDCS aMEP $(p=0.018)$.

\section{DISCUSSION}

Our results provide first evidence for a smoking-induced restitution of impaired LTD-like neuroplasticity following
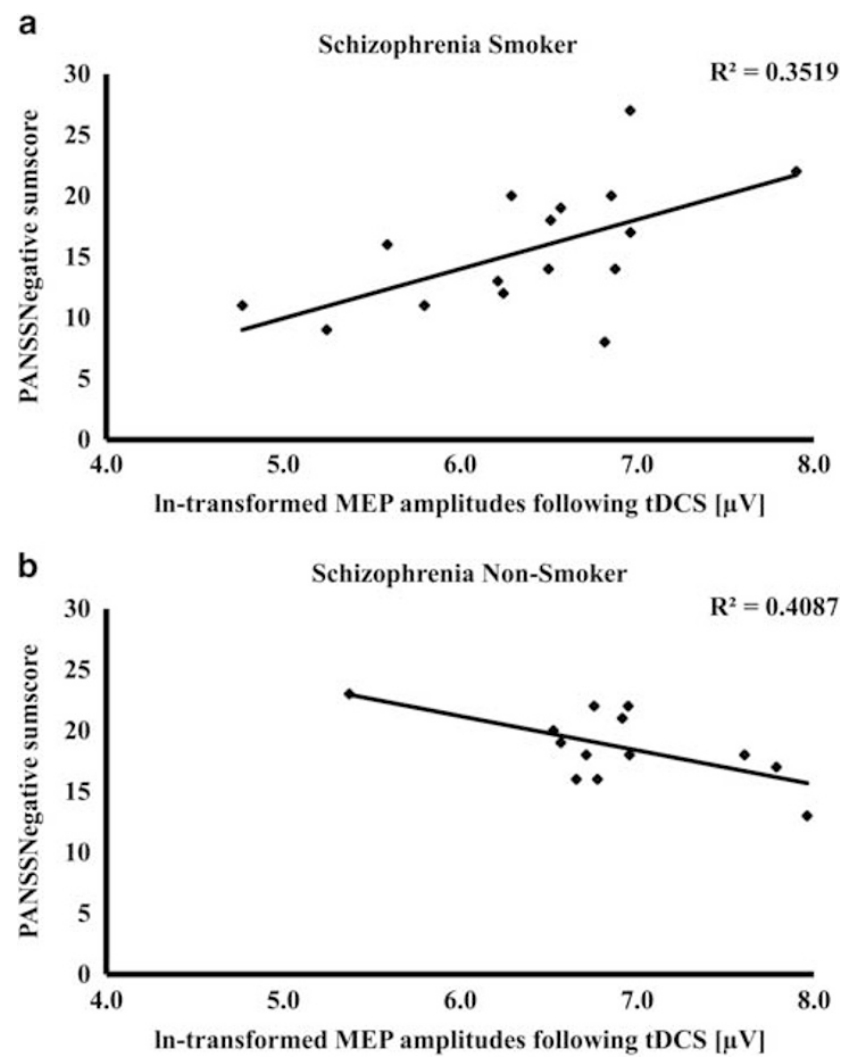

Figure $\mathbf{2}$ Correlations between the post-MEP values and negative symptoms. Smoking schizophrenia patients (a) showed a significant positive correlation between PANSS Negative and post-MEP sizes $(p=0.012)$, whereas nonsmoking schizophrenia patients (b) showed a negative correlation between PANSS Negative and post-MEP sizes $(p=0.019)$. cathodal tDCS in schizophrenia patients. Nonsmoking schizophrenia patients displayed abolished LTD-like plasticity compared with both smoking schizophrenia patients and respective healthy controls. In smoking schizophrenia patients, these plasticity deficits were not present. These findings implicate a beneficial effect of nicotine on impaired neuroplasticity in schizophrenia and may contribute to the excessive smoking rates in this population.

\section{Smoking Effect on Cortical Excitability}

Foregoing investigations of motor-cortex excitability with TMS have shown a reduction in intracortical facilitation, prolonged CSP durations, enhanced short-afferent inhibition (SAI), and reduced aMEPs, indicating cortical hyopexcitability in chronic healthy smokers during nicotine withdrawal (Grundey et al, 2013; Lang et al, 2008). However, the duration of nicotine withdrawal differed between the studies, ranging from one (Lang et al, 2008) to six (Grundey et al, 2013) hours. Our baseline analyses showed no significant differences across all four study groups for RMT, CSP, and aMEPs, but the comparability to the foregoing studies is reduced, as we did not investigate paired-pulse TMS paradigms or short-afferent inhibition in all the participants. Therefore, despite being in the time range of nicotine withdrawal (at least half an hour in our sample), we could not replicate the CSP and aMEP findings described in the respective foregoing publication (Lang et al, 2008). Interestingly, we found a significant negative correlation between Fagerstroem values and CSP duration in healthy smokers, indicating at least a gradual impact of smoking on CSP duration. Reviewing the literature for motor-cortical excitability in schizophrenia (Bunse et al, 2014), general differences between schizophrenia patients and healthy controls for CSP are subject to a large inter-study variability, possibly influenced by the smoking status of the study subjects, which is usually not reported in these studies. Prolonged, shortened, or unchanged CSP durations in schizophrenia patients were explained by different disease states and different medications (Bunse et al, 2014).

\section{Smoking Effect on tDCS-Induced Neuroplasticity in Healthy Subjects}

In our study, healthy nonsmokers displayed a significant reduction in MEP amplitudes following cathodal tDCS. In smokers, only a trend-level MEP reduction was obtained. Independent $t$-tests did not show significant differences between healthy smokers and nonsmokers for MEP amplitudes before and after stimulation. These findings are in line with data previously reported for healthy nonsmokers, which displayed a significant MEP reduction following cathodal tDCS in the respective placebo conditions of these drug trials (Grundey et al, 2012a; Thirugnanasambandam et al, 2011). In the intervention groups receiving either prolonged nicotine patch application (Thirugnanasambandam et al, 2011) or fast-acting nicotine nasal spray application (Grundey et al, 2012a), the results of these studies showed a reduced or abolished LTD-like neuroplasticity response following cathodal tDCS.

With regard to our findings in healthy smokers, one comparable study showed a significant MEP reduction 
following cathodal tDCS in healthy subjects under nicotine withdrawal for $10 \mathrm{~h}$, but only a trendwise MEP reduction following nicotine patch application (Grundey et al, 2012b). As in our experimental setup, only a short period of nicotine withdrawal preceded the experiments and these subjects displayed related LTD-like plasticity responses, as did healthy subjects following prolonged nicotine patch application (Grundey et al, 2012b), so one might argue that our withdrawal duration may be insufficient to restore nicotine-related abolishment of plasticity. The effects in smokers might therefore be at least partially determined by an impact of nicotine. These pharmacological challenges in healthy smokers and nonsmokers suggest that, dependent on pharmacokinetic properties and the number and density of cerebral nAChR (Mukhin et al, 2008), nicotine has a complex but prominent impact on cortical plasticity

\section{Nicotine-Induced Plasticity Restoration in Patients with Schizophrenia}

In nonsmoking schizophrenia patients, cathodal tDCS failed to induce LTD-like plasticity, which is consistent with previously described dysfunctional neuroplasticity in schizophrenia patients following tDCS and other noninvasive brain stimulation techniques (Hasan et al, 2013a). In contrast, smoking schizophrenia patients showed a clear LTD-like plasticity response that was even similar to that observed in the respective healthy controls. This questions the current knowledge regarding general plasticity deficits in schizophrenia patients following any kind of noninvasive brain stimulation (Hasan et al, 2013a) by proposing that LTD-like plasticity can also be induced in schizophrenia patients depending on their smoking status.

The interpretation of these novel findings is complex because various underlying mechanisms may contribute to plasticity deficits in schizophrenia. Apart from the effect of the disease itself, foregoing studies have identified antipsychotic treatment as a major contributor to reduced cortical excitability in schizophrenia patients (Liu et al, 2009; Daskalakis et al, 2008b) and one could only speculate in which way and to what extent chronic smoking interacts with different transmitter systems. Our findings may not only be explained by mechanisms involving pre- and postsynaptic cholinergic, glutamatergic and GABAergic neurotransmission leading to complex interactions between inhibition and facilitation of pyramidal neurons (Alkondon et al, 2000; Dani, 2001), but also by structural brain changes. Chronic smoking induces long-lasting modifications of cortical architecture affecting dendritic arborization and nicotinic receptor density (Breese et al, 1997; Gonzalez et al, 2005).

Different lines of evidence contribute to the concept of disturbed neuroplasticity in schizophrenia. Both hypo- and hyperfunction of glutamatergic $N$-methyl-d-Aspartate (NMDA) receptors have been supposed to result in impaired neuroplasticity ( $\mathrm{Paz}$ et al, 2008). This glutamatergic dysfunction has also been linked to alterations in GABAergic neurotransmission, as a reduced density of GABAergic interneurons that express certain NMDAR subunits has been shown in neuropathological investigations (Woo et al, 2004). Reduced inhibition in schizophrenia has been linked to a substantial decrease in GABAergic interneurons, the
GABA-synthesizing enzyme glutamic acid decarboxylase (GAD67), and GABA-related gene expression in various parts of the schizophrenia brain (Benes, 2011; Hashimoto et al, 2008b). Adding to the complexity of neuroplasticity regulation, the transmitter release and receptor expression of NMDA and GABA have both been shown to be modulated through cholinergic presynaptic projections (Wang et al, 2007) that increase GABAergic input towards pyramidal cells (McGehee, 2007). Given the influence of nicotine and acetylcholine on the regulation of cortical excitability functions (Alkondon et al, 2000), one could assume that the associations between impaired glutamatergic, GABAergic, and cholinergic neurotransmission might result in an imbalance between facilitation and inhibition tilted toward disinhibition and excessive excitatory pruning, as discussed as one important pathophysiological feature of schizophrenia (Rapoport et al, 2012).

Alternatively, assuming a neuronal effect of nicotine after half an hour withdrawal, the impact of nicotine on calcium influx via $\alpha 7$ - and $\alpha 4 \beta 2$ receptors together with tDCSinduced NMDA-receptor activation would shift plasticity to the so-called 'no mans land' (Lisman, 2001) in healthy smokers. In nonsmoking schizophrenia patients, one could speculate that the well-established NMDA-receptor hypoactivity (Javitt et al, 2012) would not be sufficient to induce LTD-like plasticity, but a nicotine-induced increase in calcium, as outlined above, would add sufficient calcium to induce LTD-like neuroplasticity in smoking schizophrenia patients.

\section{Functional Implications}

Concerning the hereby-entailed consequences for neuroplasticity in patients with schizophrenia, different functional consequences have to be taken into account. A reduced signal-to-noise ratio with a consecutive reduced filter-function has been proposed to explain cognitive and memory deficits in schizophrenia (Rolls et al, 2008). Reviewing the available literature for cortical excitability and plasticity in schizophrenia using noninvasive brain stimulation, a clear picture emerges. Reduced cortical inhibition (eg, reduced short-interval intracortical inhibition, for review see (Bunse et al, 2014)) might cause enhanced cortical noise, leading to reduced spike-timing-dependent plasticity (as measured by paired-associative stimulation) (Frantseva et al, 2008) and reduced nonfocal plasticity (as measured by anodal and cathodal tDCS) via enhanced asynchronous baseline cortical activity (Hasan et al, 2012b; Hasan et al, 2011)

Our observed effect of enhanced LTD-like plasticity associated with nicotine intake through chronic smoking might reduce cortical noise and thus increase the signal-tonoise ratio in schizophrenia patients. This would be in line with the observation that LTD has an important role in hippocampal-based learning and memory functions (Collingridge et al, 2010), suggesting a direct contribution of LTD to information storage (Kemp and ManahanVaughan, 2007). In accordance, a noise-reducing effect of cathodal tDCS was suggested in previous experiments (Antal et al, 2004). Evidence from functional imaging (Winterer et al, 2006), as well as from neurophysiological studies (Hasan et al, 2013a), has linked the concept of a 
reduced signal-to-noise ratio to the observation of prefrontal microcircuitry dysfunction in patients with schizophrenia, which is thought to be of key relevance for attention and working memory deficits, as both cognitive processes appear to be conveyed in particular by the prefrontal cortex (PFC; Levin, 1992). Regarding the involvement of the cholinergic system and the potential influence of nicotine, evidence points to the notion that neuroplasticity in the PFC is mediated substantially through nAChR activity (McGehee, 2007). Furthermore, it was shown that nicotine intake can improve the cortical signal-to-noise ratio by enhancing GABAergic neurotransmission (Couey et al, 2007).

Thus, chronic smoking might enhance the signal-to-noise ratio via restitution of LTD-like neuroplasticity by enhancing GABAergic neurotransmission or calcium influx in schizophrenia patients and thereby improve functions. Interestingly, the smoking schizophrenia patients had less negative symptoms compared with the nonsmoking patients. Moreover, in smoking schizophrenia patients, negative symptoms correlated positively with post-tDCS MEP sizes, whereas nonsmoking schizophrenia patients showed the opposite effect. This indicates that enhanced LTD-like plasticity in smoking schizophrenia patients is related to reduced negative symptoms.

\section{Limitations}

For a comprehensive interpretation of our results, some important limitations have to be taken into account. The first limitation of this study relates to the experimental design, which had to be adapted to the procedure tolerance of schizophrenia patients and thus is shorter than investigations in healthy controls. Furthermore, data of a comparative sample receiving sham tDCS were not available to correct for a putative bias related to regression to the mean in the schizophrenia group following stimulation. Second, we did not recruit patients consecutively, but integrated data from three projects (Hasan et al, 2012a; Hasan et al, 2013b; Hasan et al, 2012b) to answer this specific research question. Third, we neither tested anodal tDCS nor different PAS protocols, which would be important to specifically address the functional consequences of chronic smoking with regard to the focusing effect of nicotine. The fourth limitation is that the duration of nicotine withdrawal of at least half an hour in our study differed from the $10 \mathrm{~h}$ in some foregoing study protocols. Hence, the comparability of our data for healthy subjects to previous research is restricted. As we cannot rule out differences in the timing of the last cigarette between smoking schizophrenia and healthy subjects and as the degree of nicotine dependence is different between these groups, both factors have to be considered as potential confounding variables. One should also note that many different compounds in tobacco smoke aside from nicotine could account for some of the observed effects. As no pharmacological administration of pure nicotine was performed, conclusions as to what extent other tobacco smoke compounds might contribute to neuroplasticity remain elusive. Future studies need to clarify the effects of nicotine on cortical plasticity in schizophrenia using, eg, nicotine transdermal patches agents or nasal spray.
Furthermore, we did not obtain blood levels or breath CO concentration to verify compliance of smoking subjects. A further limitation is that schizophrenia patients were on a stable antipsychotic treatment and we cannot rule out an impact of antipsychotics on cortical plasticity or specifically test the interaction between antipsychotics, smoking, and cortical plasticity. Finally, though being within the range of other studies in the field, our sample size is rather small, meaning that some results would not survive correction for multiple testing.

\section{Summary and Outlook}

Our study shows that chronic smoking restores reduced LTD-like plasticity following cathodal tDCS in schizophrenia patients and provides a new biological explanation for the increased rates of nicotine dependency in this population. Furthermore, our findings contribute to the notion that nicotine intake through smoking might stabilize the impaired inhibition-facilitation balance in the schizophrenic brain and thus helps to increase a pathologically reduced signal-to-noise ratio. These network effects might be relevant for reducing negative symptoms and could explain the cognitive enhancing effect of smoking in schizophrenia. As the effects of smoking on cortical plasticity were not taken into account in the past, future studies need to investigate the impact of nicotine to allow an in-depth understanding of impaired neuroplasticity in schizophrenia. With regard to the future development of nicotinergic drugs, our study delivers additional support for an important role of nicotinergic stimulation for the treatment of schizophrenia.

\section{FUNDING AND DISCLOSURE}

The authors declare that, except income received from primary employers, no financial support or compensation, apart from the acknowledged grant to MA Nitsche, has been received from any individual or corporate entity over the past 3 years for research or professional service and there are no personal finance holdings that could be perceived as constituting a potential conflict of interest. However, other conflicts of interests not related to this publication are: $\mathrm{T}$ Wobrock was the honorary speaker for Alpine Biomed, AstraZeneca, Cerebromed, Bristol-Myers-Squibb, Eli Lilly, I3G, Janssen-Cilag, Novartis, Lundbeck, Sanofi-Aventis, Otsuka, and Pfizer, and has accepted travel or hospitality not related to a speaking engagement from AstraZeneca, Bristol-Myers-Squibb, Eli Lilly, Janssen-Cilag, and SanofiSynthelabo; he is a member of the advisory board of Janssen-Cilag and has received a research grant from AstraZeneca, I3G, and AOK (health insurance company). MA Nitsche is a member of advisory boards of Neuroelectronics, UCB, and Eisai, and was the honorary speaker for UCB, Eisai, and Glaxo-SmithKline. P Falkai was the honorary speaker for Janssen-Cilag, AstraZeneca, Eli Lilly, Bristol-Myers-Squibb, Lundbeck, Pfizer, Bayer Vital, SmithKline Beecham, Wyeth, and Essex. During the last 5 years, but not presently, he was a member of the advisory boards of Janssen-Cilag, AstraZeneca, Eli Lilly, and Lundbeck. A Hasan has been invited to scientific meetings by Lundbeck, Janssen-Cilag, and Pfizer, and he received 
paid speakership by Desitin and BAK. He is a member of the Roche Advisory Board. All the other authors report no conflict of interest.

\section{ACKNOWLEDGEMENTS}

MA Nitsche is supported by DFG grant NI 683/4-2. We would like to thank Ms Louise Marshall (UCL, Institute of Neurology) for English editing.

\section{REFERENCES}

Aleisa AM, Alzoubi KH, Alkadhi KA (2006). Nicotine prevents stress-induced enhancement of long-term depression in hippocampal area CA1: electrophysiological and molecular studies. J Neurosci Res 83: 309-317.

Alkondon M, Pereira EF, Almeida LE, Randall WR, Albuquerque EX (2000). Nicotine at concentrations found in cigarette smokers activates and desensitizes nicotinic acetylcholine receptors in CA1 interneurons of rat hippocampus. Neuropharmacology 39: 2726-2739.

Antal A, Nitsche MA, Kruse W, Kincses TZ, Hoffmann KP, Paulus W (2004). Direct current stimulation over V5 enhances visuomotor coordination by improving motion perception in humans. J Cogn Neurosci 16: 521-527.

Benes FM (2011). Regulation of cell cycle and DNA repair in postmitotic GABA neurons in psychotic disorders. Neuropharmacology 60: $1232-1242$.

Breese CR, Marks MJ, Logel J, Adams CE, Sullivan B, Collins AC et al (1997). Effect of smoking history on [3H]nicotine binding in human postmortem brain. J Pharmacol Exp Ther 282: 7-13.

Bunse T, Wobrock T, Strube W, Padberg F, Palm U, Falkai P et al (2014). Motor cortical excitability assessed by transcranial magnetic stimulation in psychiatric disorders: a systematic review. Brain Stimul 7: 158-169.

Collingridge GL, Peineau S, Howland JG, Wang YT (2010). Longterm depression in the CNS. Nat Rev Neurosci 11: 459-473.

Couey JJ, Meredith RM, Spijker S, Poorthuis RB, Smit AB, Brussaard $\mathrm{AB}$ et al (2007). Distributed network actions by nicotine increase the threshold for spike-timing-dependent plasticity in prefrontal cortex. Neuron 54: 73-87.

D’Souza MS, Markou A (2012). Schizophrenia and tobacco smoking comorbidity: nAChR agonists in the treatment of schizophrenia-associated cognitive deficits. Neuropharmacology 62: 1564-1573.

Daskalakis ZJ, Christensen BK, Fitzgerald PB, Chen R (2008a). Dysfunctional neural plasticity in patients with schizophrenia. Arch Gen Psychiatry 65: 378-385.

Daskalakis ZJ, Christensen BK, Fitzgerald PB, Moller B, Fountain SI, Chen R (2008b). Increased cortical inhibition in persons with schizophrenia treated with clozapine. J Psychopharmacol 22: 203-209.

Dani JA (2001). Overview of nicotinic receptors and their roles in the central nervous system. Biol Psychiatry 49: 166-174.

Dome P, Lazary J, Kalapos MP, Rihmer Z (2010). Smoking, nicotine and neuropsychiatric disorders. Neurosci Biobehav Rev 34: $295-342$.

Frantseva MV, Fitzgerald PB, Chen R, Moller B, Daigle M, Daskalakis ZJ (2008). Evidence for impaired long-term potentiation in schizophrenia and its relationship to motor skill learning. Cereb Cortex 18: 990-996.

Gonzalez CL, Gharbawie OA, Whishaw IQ, Kolb B (2005). Nicotine stimulates dendritic arborization in motor cortex and improves concurrent motor skill but impairs subsequent motor learning. Synapse 55: 183-191.
Grundey J, Thirugnanasambandam N, Kaminsky K, Drees A, Skwirba AC, Lang N et al (2012a). Neuroplasticity in cigarette smokers is altered under withdrawal and partially restituted by nicotine exposition. J Neurosci 32: 4156-4162.

Grundey J, Freznosa S, Klinker F, Lang N, Paulus W, Nitsche MA (2013). Cortical excitability in smoking and not smoking individuals with and without nicotine. Psychopharmacology (Berl) 229: 653-664.

Grundey J, Thirugnanasambandam N, Kaminsky K, Drees A, Skwirba AC, Lang N et al (2012b). Rapid effect of nicotine intake on neuroplasticity in non-smoking humans. Front Pharmacol 3: 186.

Hasan A, Wobrock T, Rajji T, Malchow B, Daskalakis ZJ (2013a). Modulating neural plasticity with non-invasive brain stimulation in schizophrenia. Eur Arch Psychiatry Clin Neurosci 263: 621-631.

Hasan A, Misewitsch K, Nitsche MA, Gruber O, Padberg F, Falkai P et al (2013b). Impaired motor cortex responses in non-psychotic first-degree relatives of schizophrenia patients: a cathodal tDCS pilot study. Brain Stimul 6: 821-829.

Hasan A, Aborowa R, Nitsche MA, Marshall L, Schmitt A, Gruber O et al (2012a). Abnormal bihemispheric responses in schizophrenia patients following cathodal transcranial direct stimulation. Eur Arch Psychiatry Clin Neurosci 262: 415-423.

Hasan A, Nitsche MA, Herrmann M, Schneider-Axmann T, Marshall L, Gruber O et al (2012b). Impaired long-term depression in schizophrenia: a cathodal tDCS pilot study. Brain Stimul 5: 475-483.

Hasan A, Nitsche MA, Rein B, Schneider-Axmann T, Guse B, Gruber O et al (2011). Dysfunctional long-term potentiation-like plasticity in schizophrenia revealed by transcranial direct current stimulation. Behav Brain Res 224: 15-22.

Hashimoto K, Ishima T, Fujita Y, Matsuo M, Kobashi T, Takahagi M et al (2008a). Phencyclidine-induced cognitive deficits in mice are improved by subsequent subchronic administration of the novel selective alpha7 nicotinic receptor agonist SSR180711. Biol Psychiatry 63: 92-97.

Hashimoto T, Bazmi HH, Mirnics K, Wu Q, Sampson AR, Lewis DA (2008b). Conserved regional patterns of GABA-related transcript expression in the neocortex of subjects with schizophrenia. Am J Psychiatry 165: 479-489.

Huerta PT, Lisman JE (1995). Bidirectional synaptic plasticity induced by a single burst during cholinergic theta oscillation in CA1 in vitro. Neuron 15: 1053-1063.

Jacobsen LK, D'Souza DC, Mencl WE, Pugh KR, Skudlarski P, Krystal JH (2004). Nicotine effects on brain function and functional connectivity in schizophrenia. Biol Psychiatry 55: 850-858.

Javitt DC, Zukin SR, Heresco-Levy U, Umbricht D (2012). Has an angel shown the way? Etiological and therapeutic implications of the PCP/NMDA model of schizophrenia. Schizophr Bull 38: 958-966.

Kemp A, Manahan-Vaughan D (2007). Hippocampal long-term depression: master or minion in declarative memory processes? Trends Neurosci 30: 111-118.

Lang N, Hasan A, Sueske E, Paulus W, Nitsche MA (2008). Cortical hypoexcitability in chronic smokers? A transcranial magnetic stimulation study. Neuropsychopharmacology 33: 2517-2523.

Levin ED (1992). Nicotinic systems and cognitive function. Psychopharmacology (Berl) 108: 417-431.

Levin ED, McClernon FJ, Rezvani AH (2006). Nicotinic effects on cognitive function: behavioral characterization, pharmacological specification, and anatomic localization. Psychopharmacology (Berl) 184: 523-539.

Lisman JE (2001). Three Ca2 + levels affect plasticity differently: the LTP zone, the LTD zone and no man's land. J Physiol 532: 285.

Liu SK, Fitzgerald PB, Daigle M, Chen R, Daskalakis ZJ (2009). The relationship between cortical inhibition, antipsychotic treatment, and the symptoms of schizophrenia. Biol Psychiatry 65: 503-509. 
Martin-Ruiz CM, Haroutunian VH, Long P, Young AH, Davis KL, Perry EK et al (2003). Dementia rating and nicotinic receptor expression in the prefrontal cortex in schizophrenia. Biol Psychiatry 54: 1222-1233.

McGehee DS (2007). Nicotine and synaptic plasticity in prefrontal cortex. Sci STKE 2007: pe44.

Mukhin AG, Kimes AS, Chefer SI, Matochik JA, Contoreggi CS, Horti AG et al (2008). Greater nicotinic acetylcholine receptor density in smokers than in nonsmokers: a PET study with 2-18FFA-85380. J Nucl Med 49: 1628-1635.

Nelson CL, Burk JA, Bruno JP, Sarter M (2002). Effects of acute and repeated systemic administration of ketamine on prefrontal acetylcholine release and sustained attention performance in rats. Psychopharmacology (Berl) 161: 168-179.

Nakauchi S, Sumikawa K (2014). Endogenous ACh suppresses LTD induction and nicotine relieves the suppression via different nicotinic ACh receptor subtypes in the mouse hippocampus. Life Sci 111: 62-68.

Nitsche MA, Cohen LG, Wassermann EM, Priori A, Lang N, Antal A et al (2008). Transcranial direct current stimulation: State of the art 2008. Brain Stimul 1: 206-223.

Oldfield RC (1971). The assessment and analysis of handedness: the Edinburgh inventory. Neuropsychologia 9: 97-113.

Oxley T, Fitzgerald PB, Brown TL, de Castella A, Daskalakis ZJ, Kulkarni J (2004). Repetitive transcranial magnetic stimulation reveals abnormal plastic response to premotor cortex stimulation in schizophrenia. Biol Psych 56: 628-633.

Paz RD, Tardito S, Atzori M, Tseng KY (2008). Glutamatergic dysfunction in schizophrenia: from basic neuroscience to clinical psychopharmacology. Eur Neuropsychopharmacol 18: 773-786.

Rapoport JL, Giedd JN, Gogtay N (2012). Neurodevelopmental model of schizophrenia: update 2012. Mol Psychiatry 17: 1228-1238.

Rolls ET, Loh M, Deco G, Winterer G (2008). Computational models of schizophrenia and dopamine modulation in the prefrontal cortex. Nat Rev Neurosci 9: 696-709.

Sacco KA, Termine A, Seyal A, Dudas MM, Vessicchio JC, Krishnan-Sarin S et al (2005). Effects of cigarette smoking on spatial working memory and attentional deficits in schizophrenia: involvement of nicotinic receptor mechanisms. Arch Gen Psychiatry 62: 649-659.

Thirugnanasambandam N, Grundey J, Adam K, Drees A, Skwirba AC, Lang N et al (2011). Nicotinergic impact on focal and nonfocal neuroplasticity induced by non-invasive brain stimulation in non-smoking humans. Neuropsychopharmacology 36: 879-886.

Wang F, Chen H, Steketee JD, Sharp BM (2007). Upregulation of ionotropic glutamate receptor subunits within specific mesocorticolimbic regions during chronic nicotine self-administration. Neuropsychopharmacology 32: 103-109.

Winterer G, Musso F, Beckmann C, Mattay V, Egan MF, Jones DW et al (2006). Instability of prefrontal signal processing in schizophrenia. Am J Psychiatry 163: 1960-1968.

Woo TU, Walsh JP, Benes FM (2004). Density of glutamic acid decarboxylase 67 messenger RNA-containing neurons that express the $\mathrm{N}$-methyl-D-aspartate receptor subunit NR2A in the anterior cingulate cortex in schizophrenia and bipolar disorder. Arch Gen Psychiatry 61: 649-657.

Supplementary Information accompanies the paper on the Neuropsychopharmacology website (http://www.nature.com/npp) 